\title{
Recurrent Afebrile Episodes of Spontaneous Pneumothorax in a Child with Well Controlled Systemic Lupus Erythematosus Disease
}

\section{Mehta $\mathbf{S}^{1}$}

${ }^{1}$ Dr. Sudhir Mehta MBBS, DCH, DNB (Paediatrics), MRCPCH, Department of Paediatrics, Bhandari Hospital and Research Center, Indore, Madhya Pradesh, India.

Address for correspondence:

Dr. Sudhir Mehta

E-mail: samsudhir123@yahoo.co.in

\section{How to cite}

Mehta S. Recurrent Afebrile Episodes of Spontaneous Pneumothorax in a Child with Well Controlled Systemic Lupus Erythematosus Disease. J Nepal Paediatr Soc 2016;36(1):9496.

doi: http://dx.doi.org/10.3126/jnps.v36i1.12419

This work is licensed under a Creative Commons Attribution 3.0 License.

\section{(c) (i)}

\begin{abstract}
Systemic Lupus Erythematosus is an autoimmune disorder with multiple system involvement. Despite its pleuro-pulmonary involvement, recurrent spontaneous pneumothoraces are rare complication of childhood SLE. We report a 12 year old girl having SLE with nephritis, who developed recurrent episodes of spontaneous pneumothoraces. Surgical management with prolonged evacuation and aggressive immunosuppressive therapy improves the outcome for these patients.
\end{abstract}

Key words: Recurrent, Pneumothorax, Systemic Lupus Erythematosus, Contributory factors

\section{Introduction}

SLE is a chronic systemic disease, which can involve many organs like kidney, skin, and brain. Involvement of the lung is common in adult patients with SLE, with more than half of adult patients having lung involvement at any time during the illness. However it has infrequently been reported in children with SLE. A number of pulmonary complications including pleuritis, infectious pneumonia, pulmonary hemorrhage, pulmonary hypertension and pneumothorax are known to occur in adult SLE. Of these well known complications, spontaneous pneumothorax is rare to occur and is not reported in pediatric patients. Respiratory involvement can result primarily from inflammatory process of SLE or secondarily from complication of SLE nephritis or due to infections. The outcome of spontaneous pneumothorax in SLE patients is dismal.

\section{The Case}

Twelve year old female child, a diagnosed case of SLE presented with recurrent episodes of spontaneous pneumothorax in form of cough and respiratory distress for last two years. She was diagnosed four years back, when she presented with symptoms of mild fever, arthlagia, anaemia, oral ulceration, photosensitivity associated with hypertension. There was no history suggestive of the involvement of pulmonary system at that time. Her hemogram was suggested of microcytic hypochromic anaemia. Her urine analysis had microscopic haematuria and albuminuria of 2+ to $3+$ by dipstick with cellular casts. Antinuclear antibody (ANA) as well as Anti dsDNA 
done by immunoflourescence were both positive. She met the American College of Rheumatology 1997 Revised Classification Criteria for SLE. She was treated with steroids and antihypertensives. In view of features of nephritis, renal biopsy was done which revealed diffuse proliferative glomerulonephritis suggestive of class V SLE nephritis by the criteria of the International Society of Nephrology. She was started on $\mathrm{NIH}$ protocol for management of SLE nephritis ${ }^{1}$ and was on regular follow up. Since last two years, she had five afebrile episodes sudden respiratory distress with clinical features and chest $X$ ray finding suggestive of pneumothorax. This time she presented in paediatric emergency with complains of sudden onset of cough and severe respiratory distress without fever. On examination she was sick looking and in distress. She was afebrile with PR of $104 / \mathrm{min}, \mathrm{RR}$ of $44 / \mathrm{min}$ and BP of 130/86 mm of $\mathrm{Hg}$. Examination of chest revealed no air entry on right side with hyper resonance on percussion. Rest of systemic examination was normal. CXR (Figure 1) showed right sided pneumothorax with collapse of underlying lung with no evidence of pneumonia and CBC revealed anaemia with $\mathrm{Hb}$ of $8.2 \mathrm{mg} / \mathrm{dl}, 6700$ WBCs (P54\%, L44\%, B1\%, E1\%) and platelets of 1.69 lakhs with microcytic hypochromic anaemia. Immediate chest tube insertion and pneumothorax evacuation was carried out. Chest tube was kept in situ until she improved and chest $X$ ray revealed expansion of lung (Figure 2). Finally she was discharged from hospital after 1 week following complete recovery from pneumothorax. She is on regular treatment with good compliance. She is doing well without any worsening except for recurrent episodes of spontaneous pneumothorax.

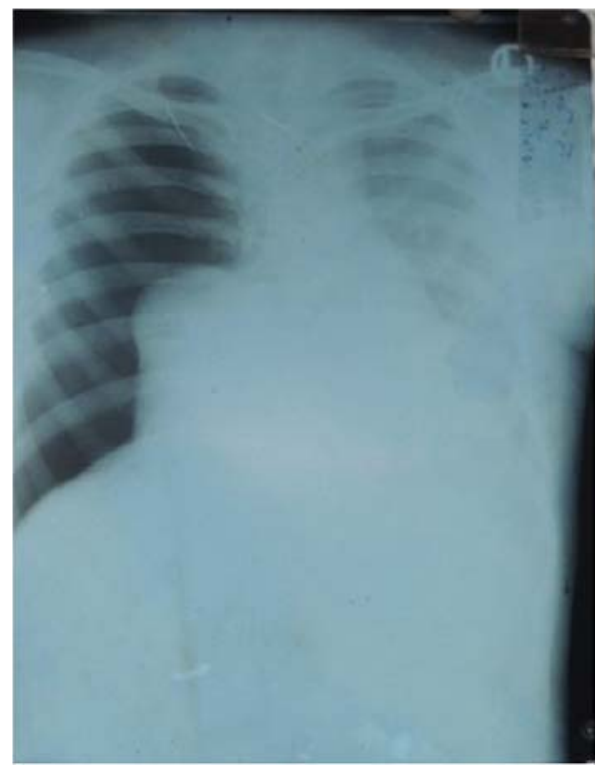

Fig 1: CXR showing Right sided pneumothorax

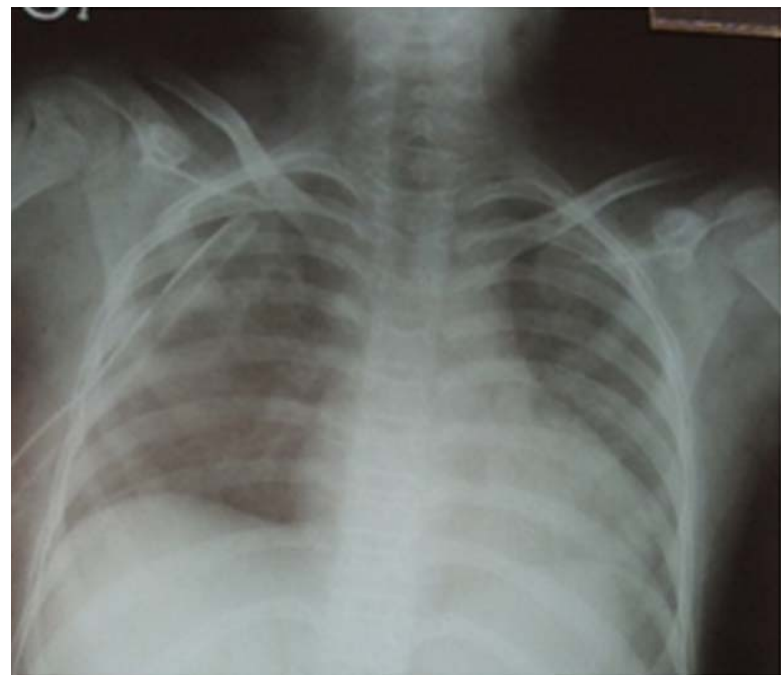

Fig 2: CXR showing disappearance of pneumothorax after ICD insertion.

\section{Discussion}

SLE is a non- organ specific connective tissue disease which involves many organs like kidney, skin and brain. The lungs are yet another organ that may be involved. It has been reported that pleuropulmonary manifestations are seen in approximately $50-70 \%$ of adult patients with $\mathrm{SLE}^{2,3}$. Although pulmonary involvement in SLE has rarely been systematically investigated in children, pulmonary involvement is reported to be 5 to $67 \%$ in few studies ${ }^{4,5,6}$. All parts of pulmonary system can be involved including parenchyma, vessels, pleura, airway and even neuromuscular control of respiration. A number of pulmonary complications known to be associated with adult SLE like pleurisy, pneumonitis, pulmonary haemorrhage, pulmonary hypertension, diffuse interstitial disease, and shrinking lungs have infrequently been reported in paediatric SLE ${ }^{4,5,6,7}$. However pleuropulmonary infections and subclinical lung disease have been reported in childhood-onset $\mathrm{SLE}^{5,8}$. But out of all these complications, spontaneous pneumothorax has not been reported in paediatric SLE in literature.

A number of putative mechanisms have been presented previously. A pathological investigation in previous reports suggests that there frequently is associated involvement of pulmonary parenchyma in patients with SLE who develop pneumothorax ${ }^{9}$. It has been proposed that underlying pneumonitis predisposes to pneumothorax either by formation of macroscopic blebs or by causing microscopic involvement of pulmonary parenchyma in form of infarction ${ }^{9}$. Patients who had pulmonary function testing prior to development of pneumothorax have had distinctive 
evidence of restrictive lung disease supporting this hypothesis. But our patient had normalpulmonary function on spirometry prior to development of first episode of pneumothorax. In general glucocorticoids have antagonistic effect on growth factors and collagen deposition during wound healing, resulting in tissue fragility ${ }^{10}$. On the other hand, it has been reported that few patients with various systemic disorders, like glomerulonephritis, leukemia, Hodgkin's lymphoma etc who are treated with cyclophosphomide develop late onset lung disease complicated by pneumothorax. But this may be contributory factor as our patient, was on cyclophophamide therapy prior to pneumothorax. Review of literature ${ }^{11}$ identified total 11 cases of SLE complicated by pneumothorax in adults. 10 out of 11 had evidence of underlying parenchymal lesions including 9 who had evidence of pleurisy. 10 out of 11 patients had received glucocorticoid therapy for atleast 3 weeks before the occurrence of pneumothorax. Literature review revealed primarily four factors contributing to pneumothorax that include underlying immune process of disease, lung infections, role of glucocorticoids and cyclophosphamide therapy. Tissue fragility caused by these might contribute to pneumothorax in these patients. Our patient did not have any evidence of lung parenchymal involvement or underlying lung infection. But she had received cycles of chemotherapy with cyclophosphomide Therefore, the disease process itself contributed to the formation of pneumothorax in this case probably potentiated by the cyclophosphamide and glucocorticoid therapy. Most SLE patients with pneumothorax reported in literature succumbed to the illness. Our patient had good outcome as she had no evidence of involvement of lung parenchyma. She responded to conservative management of surgical evacuation and aggressive immunosuppressive therapy each time.

\section{Conclusion}

Recurrent episodes of spontaneous pneumothoraces in a child with known case of SLE, is a very rare complication with multiple underlying putative mechanisms. Although underlying disease process and its immunosuppressive therapy both seems to be contributory. But conservative treatment along with underlying immunosuppressive therapy should be continued during each episode.

\section{References}

1. Gaurley MF, Austin HA, Vaughan EM et al. Methyl prednisolone alone or in combination in Lupus Nephritis. A randomized controlled trial . Ann Intern Med 1996;125:549-57.

2. Haupt HM, Moore GW, Hutchins GM. The lung in systemic lupus erythematosus. Analysis of the pathologic changes in 120 patients. Am J Med 1981;71:791-98.

3. Hunninghake GW, Fauci AS. Pulmonary involvement in collagen vascular diseases. Am Rev Resp Dis 1979;119:471-503.

4. Caeiro FM, Michielson FMC, Bernstein R, Hughes $\mathrm{GR}$, Ansell BM. Systemic lupus erythematosus in childhood. Ann Rheum Dis 1981;40:325-31.

5. De Jongste JC, Neijens HJ, Duiverman EJ, Bogaard JM, Kerrebijn KF. Respiratory tract disease in systemic lupus erythematosus. Arch Dis Child 1986;61:478-83.
6. Schaller J. Lupus in childhood. Clin Rheum Dis 1982;8:219-28.

7. Delgado EA, Malleson PN, Pirie GE, Petty RE. The pulmonary manifestations of childhood onset systemic lupus erythematosus. Semin Arthritis Rheum 1990;19:285-93.

8. Cerveri I, Bruschi C, Ravelli A et al. Pulmonary function in childhood connective tissue diseases. Eur Respir J 1992;5:733-38.

9. W Michael, et al. Pneumothoraces complicating systemic lupus erythematous with nephritis. Pediatr Nephrol 2002;17:261-63

10. Wicke $C$, et al. Effects os steroids and retenoids on wound healing. Arch Surg 2000;135:1265-70.

11. Tanaka N, Kusunoki $\mathrm{Y}$, Kaneko K et al. Jpn J Clin immunol 2010;33:162-68. 